\title{
Prevalencia de la enfermedad celiaca en donantes de sangre de la Comunidad de Madrid
}

\author{
M. D. García Novo, C. Garfia ${ }^{1}$, M. D. Acuña Quirós, J. Asensio, G. Zancada², S. Barrio Gutiérrez², \\ J. Manzanares ${ }^{1}$ y J. A. Solís Herruzo ${ }^{1}$
}

Hospital Universitario Niño Jesús. ${ }^{1}$ Hospital 12 de Octubre. ${ }^{2}$ Centro de Trasfusiones de la CAM. Madrid

\section{RESUMEN}

Objetivo: conocer la prevalencia de enfermedad celiaca en la población adulta de la Comunidad de Madrid utilizando como método de despistaje los anticuerpos frente a la transglutaminasa tisular.

Población y métodos: han participado de modo voluntario 2.215 donantes de sangre. Todos ellos rellenaron una encuesta. Se determinó la IgA sérica total y los anticuerpos antitransglutaminasa tisular. A los donantes con anticuerpos positivos, se les ofreció la realización de biopsia intestinal por endoscopia. La histología de la mucosa intestinal se graduó según los criterios de Marsh.

Resultados: mediante la encuesta se identificaron tres celiacos diagnosticados previamente. Once donantes presentaban anticuerpos positivos, todos ellos asintomáticos. Cuatro rechazaron la biopsia intestinal. De los siete en los que se realizó, tres tenían atrofia vellositaria y cuatro infiltrado linfocitario con vellosidades normales. En nuestro estudio el número total de donantes con enfermedad celiaca confirmada por biopsia fue de seis, lo que supone una prevalencia del 1/370. Considerando el grado I de Marsh, la prevalencia de la enteropatía por gluten sería de 1/222. La respuesta inmunológica anómala al gluten medida por la positividad de los anticuerpos fue de $1 / 201$ que alcanza valores de $1 / 158$ si consideramos los tres celiacos diagnosticados previamente.

Conclusiones: los datos de prevalencia hallados en este estudio confirman que la enfermedad celiaca constituye un problema sanitario de primer orden, que podría justificar la instauración de un programa de despistaje universal. Se ha detectado un alto número de casos de enteritis linfocitaria lo que obliga a plantearse la actitud a seguir.

Palabras clave: Enfermedad celiaca. Anticuerpos antitransglutaminasa. Prevalencia. Enteritis linfocitaria.

Este estudio ha sido realizado mediante una ayuda de la Asociación contra la Enfermedad Celiaca de Madrid.

Recibido: $15-02-07$

Aceptado: 19-02-07.

\begin{abstract}
Objective: the aim of this study was to determine the prevalence of celiac disease among the adult population of Madrid by measuring antibodies against tissue transglutaminase as serologic screening method.

Population and methods: 2,215 subjects participated voluntarily in this study. All of them completed a clinical questionnaire. We determined the levels of total IgA and antibodies to tissue transglutaminase (tTG). An intestinal biopsy by endoscopy was proposed to all subjects who were tTG-positive. The histologic lesion was classified in accordance to Marsh.

Results: three known CD cases were identified by the questionnaire. Eleven donors with tTG positivity were detected, all of them asymptomatic. Four subjects rejected the intestinal biopsy. Seven out of 11 positive subjects consented to undergo a duodenal biopsy -3 had villous atrophy and 4 had increased intraepithelial lymphocyte counts with normal villi. In our study the number of donors with biopsy-proven $\mathrm{CD}$ was 6 , and the prevalence was $1 / 370$. If we include the subcategories of gluten sensitive enteropathy (Marsh I), the prevalence would be $1 / 222$. When we considered antibody positivity the prevalence of gluten sensitivity was 1 in 201, and it reached 1 in 158 when the three known CD cases were included.

Conclusions: data on $\mathrm{CD}$ prevalence in this study confirm that $C D$ is a first-line healthcare problem that may warrant universal screening. We detected a high number of lymphocytic enteritis cases, and thus some sort of action is mandatory.
\end{abstract}

Key words: Celiac disease. Anti-transglutaminase antibodies. Prevalence. Lymphocytic enteritis.

García Novo MD, Garfia C, Acuña Quirós MD, Asensio J, Zancada G, Barrio Gutiérrez S, Manzanares J, Solís Herruzo JA. Prevalencia de la enfermedad celiaca en donantes de sangre de la Comunidad de Madrid. Rev Esp Enferm Dig 2007; 99: 337342.

Correspondencia: C. Garfia. Servicio de Medicina del Aparato Digestivo. Hospital 12 de Octubre. Avda, Andalucía, s/n. 28041 Madrid. e-mail: cgarfia@ hotmail.com 


\section{INTRODUCCIÓN}

La enfermedad celiaca (EC) es una enteropatía mediada por linfocitos T, causada por una permanente sensibilidad al gluten en individuos genéticamente susceptibles. Cursa con una lesión característica, aunque no específica de la mucosa intestinal, que cede con la retirada del gluten de la dieta (1).

La forma clásica de la enfermedad se presenta con síntomas gastrointestinales tales como diarrea, pérdida de peso, distensión abdominal y anorexia, entre otros. Otros pacientes presentan formas clínicas oligosintomáticas o con síntomas no gastrointestinales: dermatitis herpetiforme, alteraciones del esmalte o talla baja. En ocasiones, la enfermedad existe en individuos totalmente asintomáticos, como se ha demostrado en los estudios de cribado entre los familiares de primer grado de pacientes con EC, en los que se puede encontrar una mucosa intestinal plana en ausencia de cualquier síntoma. A esta forma clínica de EC se la conoce como EC silente.

Por ello es difícil establecer la frecuencia real de esta enfermedad, ya que esta varía dependiendo de que el cribado se realice en la población general, familiares, enfermos sintomáticos o en pacientes con enfermedades asociadas a la enfermedad celiaca (2).

Los estudios realizados en población general en Europa, Estados Unidos y países de América latina han encontrado frecuencias de esta enfermedad que oscilan entre 1/100-1/300 (3-6). De estos estudios se deduce que por cada caso nuevo diagnosticado de EC existen 5-10 casos no diagnosticados por ser su presentación subclínica, atípica o silente. Estos hallazgos indican que la enteropatía por sensibilidad al gluten es alta, pero las frecuencias de diagnóstico de las diferentes formas clínicas dependen de diversas variables tales como el grado de sospecha de los médicos que trabajan en las distintas especialidades o de sus posibilidades para realizar pruebas de cribado.

Una población aparentemente sana para realizar estudios de prevalencia de enfermedad celiaca silente en la población general son los donantes de sangre. En estos basamos el presente estudio.

\section{OBJETIVO}

El objetivo primario de este estudio ha sido el de conocer la prevalencia global de la EC en la población de donantes de sangre de la Comunidad de Madrid (CAM). La EC silente la hemos detectado utilizando como método de cribado la presencia de anticuerpos frente a la transglutaminasa tisular (atTG), y la prevalencia de EC ya diagnosticada mediante una encuesta realizada a esta población.

Como objetivos secundarios de este estudio nos planteamos la identificación de signos, síntomas u otras enfermedades que estén asociadas a la EC silente.

\section{POBLACIÓN Y MÉTODOS}

\section{Población}

El cálculo de la muestra (2.100 donantes) se estimó sobre la base de una frecuencia estimada de $1 / 300$, con un error alfa de 0,05 y beta de 0,1 .

Entre el 27 de febrero de 2001 y el 25 de junio de 2001 y durante los meses de junio y julio de 2002, participaron de forma voluntaria 2.215 donantes de sangre, después de haber sido informados por escrito de los objetivos de este estudio.

El 44,12\% de los donantes eran estudiantes universitarios, el 26,47\% eran donantes en unidades de donación ambulantes en la vía pública, el 23,36\%, empleados de empresas privadas, y el $6 \%$, empleados públicos. Todos ellos con domicilio fijo en la Comunidad Autónoma de Madrid. Sexo: $1.285(58 \%)$ donantes eran varones y 930 (42\%), mujeres. La edad media era 32,2 \pm 12,4 años (rango: 18-65 años).

Todos los participantes rellenaron una encuesta sobre antecedentes familiares y personales de EC, existencia de enfermedades autoinmunes asociadas a EC, alteraciones cutáneas o mucosas y síntomas y signos digestivos sugestivos de enfermedad celiaca (Tabla I).

Aquellos que lo solicitaron pudieron ampliar la información a través del médico encargado de las extracciones. Todos los donantes que quisieron participar de forma voluntaria dieron su consentimiento por escrito.

\section{Tabla I. Encuesta}

\begin{tabular}{l}
\hline Antecedentes familiares \\
Diabetes juvenil \\
Enfermedades de la piel \\
Enfermedad celiaca \\
\hline Antecedentes personales \\
Enfermedad tiroidea \\
Diabetes juvenil \\
Déficit de lgA \\
Dermatitis herpetiforme \\
Esterilidad \\
Abortos de repetición \\
Enfermedad celiaca \\
\hline Sintomas y signos \\
Estreñimiento \\
Diarrea crónica \\
Pérdida de apetito \\
Hemorragias \\
Úlceras bucales de repetición \\
Pérdida de peso \\
Distensión abdominal \\
Deterioro del esmalte dentario \\
Adormecimiento y calambres en miembros
\end{tabular}

\section{Métodos}

Los participantes autorizaron que $5 \mathrm{cc}$ de sangre se destinaran al estudio serológico. Las muestras se congelaron a $-20{ }^{\circ} \mathrm{C}$ hasta su procesamiento. 
En todos los donantes se determinaron la inmunoglobulina $\mathrm{A}(\operatorname{Ig} \mathrm{A})$ y $\operatorname{los}$ anticuerpos antitransglutaminasa tisular (atTG).

La IgA sérica total se cuantificó mediante nefelometría $\left(\right.$ Dade-Behring $\left.{ }^{\circledR}\right)$. Si las cifras de $\operatorname{IgA}$ eran inferiores a $6,67 \mathrm{mg} / \mathrm{dl}$ se interpretó que existía un déficit de IgA, por lo que se determinaron los anticuerpos antiendomisio (EMA) de la clase IgG mediante inmunofluorescencia indirecta utilizando como sustrato esófago de mono.

Los anticuerpos antitransglutaminasa tisular (atTG) se realizaron mediante técnicas de ELISA (Celikey, Pharmacia $^{\circledR}$ ) utilizando como sustrato antigénico transglutaminasa humana recombinante. Se consideró un valor de corte positivo si eran más de $8 \mathrm{U}$ y dudoso entre 5-8 U.

A las personas con atTG positivos o EMA IgG elevados, se les ofreció la posibilidad de realizar una biopsia intestinal (BI) por endoscopia. En estos casos se tomaron al menos 3 muestras para su procesamiento. La histología de la mucosa intestinal se graduó según los criterios de Marsh (7).

Clasificación de Marsh:

- Preinfiltrativo (tipo 0).

- Infiltrativo (tipo 1).

- Infiltrativo hiperplásico (tipo 2).

- Plano destructivo (tipo 3).

- Atrofico hipoplásico (tipo 4).

Se consideró el diagnóstico de enfermedad celiaca cuando en la biopsia intestinal se encontró algún grado de atrofia vellositaria.

\section{Análisis estadístico}

El análisis estadístico de la muestra se realizó con el paquete estadístico SPSS versión 11.

\section{RESULTADOS}

Existían antecedentes familiares de enfermedad celiaca por rama paterna en dos donantes y 37 presentaban antecedentes familiares de diabetes mellitus (19 por rama materna y 18 paternos), ninguno de los cuales presentaban atTG positivos.
Por los antecedentes personales de la encuesta se identificaron 3 donantes con enfermedad celiaca diagnosticada; uno de ellos debutó con dermatitis herpetiforme diagnosticada mediante biopsia de piel y atrofia intestinal confirmada por biopsia. Los tres seguían una dieta sin gluten estricta, los atTG, en todos ellos, eran negativos. Por tanto, en esta muestra, la prevalencia de EC reconocida era de 1:738.

Otros datos detectados por la encuesta fueron: 2 diabetes insulin dependientes, 29 enfermedades tiroideas, 1 donante con deficiencia conocida de $\operatorname{IgA}, 5$ mujeres con esterilidad, 7 habían tenido abortos de repetición, 20 donantes presentaban diarrea crónica, y 24 referían anorexia. Ninguno de ellos tenía elevación de anticuerpos antitransglutaminasa.

Mediante la determinación y cuantificación de $\operatorname{IgA}$ se detectó un mieloma múltiple y 8 casos de déficit de $\operatorname{Ig} \mathrm{A}$, a estos donantes se les realizó EMA $\mathrm{IgG}$ con resultado negativo.

Once donantes presentaban anticuerpos transglutaminasa positivos con un valor medio de 50,9 U (9,1-100). Todos ellos se encontraban asintomáticos, según los datos registrados en la encuesta y confirmados por entrevista telefónica. A todos se les indicó la realización de una biopsia intestinal por endoscopia digestiva alta; cuatro donantes rechazaron la biopsia $(36,35 \%)$; el valor medio de la atTG en este grupo, fue de 53,7 U (rango 16,5-100).

A los siete restantes se les realizó biopsia intestinal con los resultados de dos donantes con atrofia vellositaria severa con hiperplasia críptica e infiltrado linfoplasmocitario (grado IIIc de Marsh). En estos, el valor medio de atTG fue de 73,8 U (21,5-100). Los otros cinco donantes tenían en la biopsia intestinal lesiones mínimas, con vellosidades normales pero con linfocitos intraepiteliales del $60-90 \%$ (enteritis linfocitaria o grado I de Marsh), el valor medio de atTG en este grupo fue de 25,05 (rango: 9,1-40,2).

Los primeros fueron diagnosticados de EC silente y tratados con dieta sin gluten. A los segundos se les mantuvo con dieta libre, bajo control clínico. Durante el seguimiento uno de ellos aumentó los valores de atTG y desarrolló un hipotiroidismo subclínico autoinmune. La biopsia intestinal realizada en ese momento, a los 6 meses de la primera, demostró atrofia vellositaria parcial e hiperplasia críptica. En ese momento se le indicó la dieta sin gluten.

Tabla II. Datos de los pacientes diagnosticados de enfermedad celiaca

\begin{tabular}{lcccll}
\hline & Sexo & Edad & atTG & Biopsia intestinal & HLA \\
\hline Paciente 1 & Varón & 54 & 49 & $\begin{array}{l}\text { Atrofia vellositaria intensa e } \\
\text { hiperplasia críptica }\end{array}$ & $\begin{array}{l}\text { A11 B8 (Bw6) DRB1*07 DQB1*0201/ } \\
\text { A24 B65 (BW6) DRB1*03 (DR17) DQB1*0202 }\end{array}$ \\
\hline Paciente 2 & Mujer & 23 & $>100$ & Atrofia vellositaria subtotal & $\begin{array}{l}\text { A1 B18 (Bw6) DRB1*03 (DR17) DQB1*0201/ } \\
\text { A2 B44 (BW4) DRB1*04 DQB1*0302 (DQ8) }\end{array}$ \\
\hline Paciente 3 & Varón & 31 & $>200$ & Atrofia vellositaria & A2 B27 (BW4) DQB1*0202 DRB1*07/ \\
& & & & B60 (BW6) DQB1*0301 (DQ7) DRB1*11 \\
\hline
\end{tabular}


Por tanto, en esta muestra, el número total de donantes con EC confirmada por biopsia fue de seis, tres con un diagnóstico previo y tres celiacos silentes, diagnosticados en el estudio; lo que da una prevalencia de 1/370. La relación EC conocida/silente en esta muestra fue de 1/1.

Si consideramos el grado I de Marsh como el primer estadio del espectro de la enteropatía por gluten la prevalencia sería de $1 / 222$.

La respuesta inmunológica anómala al gluten medida por la positividad de los atTG se ha detectado en 11 de los 2.215 donantes: prevalencia de $1 / 201$, que alcanza valores de $1 / 158$ si se consideran los 3 celiacos ya identificados previamente.

\section{DISCUSIÓN}

Desde que en 1887 S. Gee describiera la enfermedad celiaca, nuestro conocimiento sobre esta enfermedad ha variado notablemente. Inicialmente se consideraba una enfermedad de inicio pediátrico, en la que la diarrea, la malabsorción y el retraso estato-ponderal eran los síntomas de presentación. Hoy en día no es raro su diagnóstico en pacientes adultos mono-oligosintomáticos e incluso asintomáticos $(8,9)$. Esto ha sido posible gracias al desarrollo de los nuevos métodos serológicos y su empleo en el cribado de la población general. Esta extensa heterogeneidad de las manifestaciones clínicas de la EC ha justificado que se la compare con un iceberg cuya parte emergente la constituyen los casos clínicos sintomáticos y la parte sumergida los asintomáticos $(2,10,11)$. Ello justifica que la verdadera prevalencia sea difícil de establecer, si bien se estima que tanto en los Estados Unidos como en Europa afecta al 0,5-1\% de la población. Así, en España esto supondría una estimación de 450.000 pacientes celiacos (están diagnosticados solamente unos 45.000 pacientes) (12).

No obstante, las cifras obtenidas en los diversos estudios sobre la prevalencia de la EC difieren según que la población investigada sea la población general, población pediátrica, donantes de sangre, individuos con factores de riesgo o enfermos sintomáticos. La metodología empleada para su cribado hace variar también los resultados: determinación de anticuerpos antigliadina (AGA), anti-endomisio (EMA), antitransglutaminasa (atTG).

Nuestro estudio fue realizado en donantes de sangre como muestra de la población sana y asintomática para explorar la base sumergida del iceberg. Sin embargo, esta población tiene un sesgo tanto a la baja como a la alta. Del estudio se excluyen aquellas personas que presentan elevación de las transaminasas, lo cual puede ser la única manifestación de la EC en adultos. Esta enfermedad es responsable del 2,7 al 5\% de las hipertransaminasemias de origen desconocido (13-15). Otro grupo que queda excluido en los estudios de donantes son las personas con anemia ferropénica refractaria al tratamiento. En este sentido se estima que el 5-6\% de los enfermos celiacos adultos asintomáticos, presentan anemia refractaria como único signo de EC (16). Por otro lado, los donantes con sintomatología digestiva u otras enfermedades es más probable que accedan a ser incluidos en el estudio.

En todos los estudios de cribado de la enfermedad se comienza con un método no invasivo: la determinación de los distintos anticuerpos dirigidos contra la gliadina, endomisio y trasglutaminasa, determinaciones que han ido variando a lo largo del tiempo demostrando una creciente sensibilidad y especificidad.

Los estudios iniciales de detección poblacional de EC utilizaron un modelo de dos niveles. Así, los realizados en 1996 en Italia por Catassi y cols. (17), dirigidos a la población infantil, realizaban inicialmente la determinación de los anticuerpos AGA IgA e IgG. Posteriormente, en los casos positivos, determinaban los anticuerpos EMA, debido a que estos son más específicos y a que los AGA tienen un nivel predictivo más bajo (18). Tras ello realizaban la biopsia intestinal en los sujetos con EMA positivos. En este estudio se encontró una prevalencia de EC global de 1/184-198.

En 1996 y 1998, se realizaron dos estudios en donantes de sangre en Suecia (19) y en EE.UU. (20) empleando la determinación de AGA y de AGA y EMA, respectivamente. La prevalencia fue de $1 / 250$, tasa inferior a la detectada en nuestro estudio si tomamos únicamente como referencia la positividad de los anticuerpos. Hovdenak y cols. (21), en Noruega, utilizando AGA IgA e IgG con posterior confirmación con EMA y biopsia en 2.096 donantes de sangre, encontraron una prevalencia de EC silente en $1 / 340$, mientras que la enfermedad sintomática en este país es de 1/675. Estos resultados son muy similares a los nuestros.

La segunda estrategia diagnóstica utilizada ha sido la determinación inicial de EMA, realizando los AGA IgG sólo en los casos de déficit de IgA, con lo que aumentaba la sensibilidad y disminuía el número de biopsias intestinales. En nuestro país, un estudio realizado por Cilleruelo y cols. (3), en 2002, en la población infantil y empleando esta estrategia junto con la biopsia intestinal mostró una prevalencia global de la EC de 1/220. Riestra y cols. (22) estudiaron la población general, en un área de Asturias mediante la determinación de AGA y/o EMA y biopsia intestinal, y encontraron una prevalencia de $1 / 389$, cifras muy similares a las obtenidas en nuestro estudio (1/370).

En los últimos años se ha difundido como método de cribado la determinación de los anticuerpos dirigidos contra la transglutaminasa humana, atTG, que es la proteína específica a la que van dirigidos los anticuerpos antiendomisio $(23,24)$. Tanto los EMA como los atTG tienen una elevada sensibilidad y especificidad, pero a favor de los atTG ya que esta es una técnica más barata, reproducible y rápida. En 2003, Mäki y cols. (25) realizaron un estudio en la población infantil de Finlandia utilizando la determinación de EMA, atTG y la biopsia intestinal encontrando una prevalencia de EC global de 1/99. Con esta misma metodología, Fassano y cols. (26) 
hallaron una prevalencia de 1/105 en la población general adulta de EE.UU. No obstante, en este trabajo, se realizó la biopsia solamente en el $30 \%$ de los casos, por lo que esta prevalencia está basada, fundamentalmente, en la serología. En 2004, Neri y cols. (27) revaloraron mediante la determinación de la atTG la población de donantes estudiados previamente en 1998 con AGA y EMA y detectaron una prevalencia de 1/125.

La alta sensibilidad y especificidad de los marcadores serológicos facilita el diagnóstico aunque, por el momento, la biopsia intestinal continúa siendo la prueba de oro. En el Consenso del NIH (National Institute Health) sobre enfermedad celiaca celebrado en junio de 2004, una de las preguntas claves fue cómo diagnosticar la enfermedad. Se concluyó que en la definición se debe hacer constar algún grado de atrofia vellositaria. Esta lesión no es patognomónica de la EC, ya que pueden observarse en otras situaciones patológicas (28). Este requisito se cumplió en 3 donantes biopsiados, por lo que la prevalencia fue del 1/370.

La presencia de linfocitos intraepiteliales es menos definitiva para hacer el diagnóstico de EC, debido a que la arquitectura es aparentemente normal. No obstante, está aceptado que la enteropatía por gluten no se restringe a la mucosa plana y existen casos en que el alto recuento de linfocitos disminuye con la dieta sin gluten y se incrementa con la reintroducción del gluten (29). Si consideramos el grado I y II de Marsh, los primeros estadios del espectro de la EC, la prevalencia en nuestro estudio aumentaría a $1 / 222$. Incluso es muy probable que esta cifra sea realmente más elevada ya que más de un tercio de los individuos con anticuerpos positivos rechazaron la biopsia intestinal. Para estos grados iniciales de la EC no existe un manejo consensuado, se puede optar: a) por determinar el HLA y realizar nuevas biopsias si existen alelos de susceptibilidad; b) controlar periódicamente al paciente; y c) como algunos autores preconizan, retirar el gluten de la dieta $(30,31)$. Argumentan para ello que, aunque hasta la fecha se consideraban asintomáticos a las personas con enteropatía grado I de Marsh, existen datos en la literatura, en series cortas de pacientes, que muestran que estos pacientes desarrollan síntomas (32) y además mejoran con la dieta. Es un hecho crucial en el diagnóstico de EC, máxime si existen anticuerpos elevados, que estos se negativizan con la dieta.

Los pacientes detectados en nuestro estudio con enteropatía de bajo grado se encontraban asintomáticos y fueron monitorizados y seguidos clínicamente. Uno de ellos desarrolló un hipotiroidismo autoinmune y atrofia vellositaria. Este caso es un ejemplo de EC latente, asintomática, poco llamativa histológicamente, que se manifestó clínicamente en el curso de su seguimiento. Por ello, estos enfermos deben ser sometidos a una vigilancia periódica con el fin de poder detectar la enfermedad en sus fases más iniciales, poder instaurar una dieta sin gluten y, de esta manera, intentar mejorar la calidad de vida, evitar el desarrollo de osteoporosis (33-35), de enfermedades autoinmunes (36), o de linfomas u otras neoplasias (37).

Dada la elevada prevalencia de la enfermedad detectada incluso en población aparentemente sana y la elevada sensibilidad y especificidad de las pruebas serológicas disponibles en la actualidad se justificaría la instauración de un programa de despistaje universal. Se ha comentado la escasa adherencia a la dieta sin gluten en pacientes asintomáticos. Los descubiertos en este trabajo permanecen con dieta sin gluten a los 2 años de este estudio.

\section{BIBLIOGRAFÍA}

1. Trier JS. Diagnosis of celiac sprue. Gastroenterology 1998; 115: 2116 .

2. Fasano A, Catasi C. Current approaches to diagnosis and treatment of celiac disease: an evolving spectrum. Gastroenterology 2001; 120 : 636-51.

3. Cilleruelo Pascual ML, Román Riechmann E, Jiménez Jiménez J, Rivero Martín MJ, Barrio Torres J, Castaño Pascual A, et al. Enfermedad celiaca silente: explorando el iceberg en población escolar. An Esp Pediatr 2002; 57: 321-6.

4. Menardo G, Brizzolara R, Bonassi S, Marchetti A, Dante GL, Pistone $\mathrm{C}$, et al. Population screening for coelic disease in a low prevalence area in Italy. Scand J Gastroenterol 2006; 41: 1414-20.

5. Oliveira RP, Sdepanian VL, Barreto JA, Cortez AJ, Carvalho FO, Bordin JO, et al. High prevalence of celiac disease in Brazilian blood donor volunteers based on screening by $\operatorname{IgA}$ antitissue transglutaminase antibody. Eur J Gastroenterol Hepatol 2007; 19: 43-9.

6. Rames Troche JM, Ramírez Iglesias MT, Rubio Tapia A, Alonso Ramos A, Velásquez A, Uscanga LF. Celiac disease could be a frequent disease in Mexico: prevalence of tissue transglutaminase antibody in healthy blood donors. J Clin Gastroenterol 2006; 40: 697700 .

7. Marsh MN. Gluten, major histocompatibility complex, and the small intestine: a molecular and immunobiologic approach to the spectrum of gluten-sensitivity ("celiac sprue"). Gastroenterology 1992; 102: 330-54.

8. Casellas F, López Vivancos J, Malagelada JR. Epidemiología actual y accesibilidad al seguimiento de la dieta de la enfermedad celiaca del adulto. Rev Esp Enferm Dig 2006; 98: 408-19.

9. Rodrigo L, Riestra S, Fuentes D, González S, López-Vázquez A, López-Larrea C. Diversas formas clínicas de presentación de la enfermedad celiaca dentro de la misma familia. Rev Esp Enferm Dig 2004; 96: 612-9.

10. Mäki M, Collin P. Coeliac disease. Lancet 1997; 349: 1755-9.

11. Kamin DS, Furuta GT. The iceberg cometh: Establishing the prevalence of celiac disease in the United States and Finland Gastroenterology 2004; 126: 359-61.

12. Rodrigo Sáez L. La enfermedad celiaca en el adulto. Rev Esp Enferm Dig 2006; 98: 397-407.

13. Volta U, De Franceschi L, Molinaro N, Zoli M, Bianchi FB. Coeliac disease hidden by cryptogenetic hypertensaminasemia. Lancet 1998; 352: 26-9.

14. Novacek G, Miehsler W, Wrba F, Ferenci P, Pender E, Vogelsang H. Prevalence and clinical importance of hypertransaminasemia in celiac disease. Eur J Gastroenterol Hepatol 1999; 11: 238-88.

15. Cuadrado A, Crespo J. Hipertransaminasemia en pacientes con negatividad de marcadores virales. Rev Esp Enferm Dig 2004; 96: 484500.

16. Carrocio A, Iannito E, Catavaio F, Montalvo G, Tumminello M, Campagna $\mathrm{P}$, et al. Sideropenic anemia and celiac disease. One study, two points of view. Dig Dis Sci 1998; 43: 673-8.

17. Catassi C, Fabiani E, Rätsch IM, Coppa GV, Giorgi PL, Pierdomenico R, et al. The coeliac iceberg in Italy. A multicentre antigliadin antibodies screening for coeliac disease in chool-age subjects. Acta Paediatr 1996; 85 (Supl. 41): 29-35.

18. Farell RJ, Kelly CP. Celiac sprue. NEJM 2002; 346: 180-8. 
19. Grodzinsky E. Screening for coeliac disease in apparently healthy blood donors. Acta Paediatr 1996; (Supl. 412): 36-8.

20. Not T, Horvath K, Hill ID, Partanen J, Hammed A, Magazzu G, et al. Celiac disease risk in the USA: Higt prevalence of antiendomysium antibodies in healthy blood donors. Scand J Gastroenterol 1998; 33 : 494-8.

21. Hovdenak N, Hovlid E, Aksnes L, Fluge G Erichsen MM, Eide J. High prevalence of asyntomatic celiac disease in Norway: a study of blood donors. Eur J Gastroenterol Hepatol 1999; 11: 185-7.

22. Riestra S, Fernández E, Rodrigo L, García S, Ocio G. Prevalence of coeliac disease in the general population of Northern Spain. Strategies of serologic screening. Scand J Gastroenterol 2000; 35: 398-402.

23. Dieterich W, Ehnis T, Bauer M, Donner P, Volta U, Riecken EO, et al. Identification of tissue transglutaminase as the autoantigen of celiac disease. Nat Med 1997; 3: 797-801.

24. Mäki M. Tissue transglutaminase as the autoantigen of coeliac disease. Gut 1997; 41: 565-6.

25. Mäki M, Mustalahti K, Kokkonen J, Kulmala P, Haapalahti M, Karttunen T, et al. Prevalence of celiac disease among children in Finland. N Engl J Med 2003; 348: 2517-24.

26. Fasano A, Berti I, Gerarduzzi T, Not T, Colletti RB, Drago S, et al. Prevalence of celiac disease in at-risk and not-at-risk groups in the United States: a large multicenter study. Arch Intern Med 2003; 163: 286-92.

27. Neri E, Not T, Horvath K, Kryszak D, Drago S, Di Pierro MR, et al. Human tissue transglutaminase ELISA and an old study: A revision of the blood donor screening study of celiac disease in the USA. Scand J Gastroenterol 2004; 39: 195-7.
28. Stephen P Jame. National Institutes of Health consensus development conference statement on celiac disease, June 28-30, 2004. Gastroenterology 2005; 128 (4 Supl. 1): S1-S9.

29. Lebenthal E, Branski D. Celiac disease: an emerging problem. J Pediatr Gastroenterol Nutr 2002; 35: 472-4.

30. Fernández Bañares F. Enfermedad celiaca del adulto: manifestaciones atípicas y diagnóstico. Rev Esp Enferm Dig 2004; 96 (Supl. II): 27-8.

31. Rosinach M, Fernández-Bañares F, Esteve Comas M, González C, Farré C, Alsina M, et al. Enfermedad celiaca (EC): ies necesario el tratamiento con dieta sin gluten (DSG) en los pacientes con enteritis linfocitaria sin atrofia vellositaria (lesión tipo Marsh I)? Gastroenterol Hepatol 2004; 27: 219

32. Wahnschaffe U, Ullrich R, Riecken EO, Schulzke JD. Celiac disease-like abnormalities in a subgroup of patients with irritable bowel síndrome. Gastroenterology 2001; 121: 1329-38.

33. American Gastroenterological Association medical position statement: celiac sprue. Gastroenterology 2001; 120: 1522-5.

34. Ciclitira PG. AGA technical review on celiac sprue. Gastroenterology $2001 ; 120$ : 1526-40.

35. West J, Logan RF, Hill PG, Lloyd A, Lewis S, Hubbard R, et al. Seroprevalence, correlates, and characteristics of undetected coeliac disease in England. Gut 2003; 52 (7): 960-5.

36. Ventura A, Magazzu G, Greco L. Duration of exposure to gluten and risk of autoimmune disorders in celiac patients. Gastroenterology 1999; 117: 303-10.

37. Conor G, Edward V. Cancer risk in celiac disease. Gastroenterology 2002; 123: 1726-9. 